\title{
Pleomorphic adenoma of minor salivary gland in the floor of mouth
}

\begin{abstract}
A 42 year old female presented to the outpatient department with a slow growing painless mass in the left side of the floor of the mouth since 6 months which had been causing some difficulty in swallowing. On examination, the mass was non-tender, firm and noncompressible with well-defined borders, pushing the left side of the tongue up, and was not bidigitally palpable. Axial and coronal views of computed tomography (CT) imaging revealed a well-circumscribed soft tissue density mass in the left floor of mouth, separate from the deep lobe of the submandibular gland. The mass was excised under general anaesthesia and histopathological examination revealed darkly stained tumour cells lying in chondromyxoid mesenchyme, suggestive of pleomorphic adenoma of a minor salivary gland.
\end{abstract}

Keywords: intraoral mass, floor of mouth, dysphagia, pleomorphic adenoma
Volume II Issue I - 2019

\author{
Ramiya Ramachandran Kaipuzha,' Davis \\ Thomas Pulimoottil ${ }^{2}$ \\ 'Department of Otorhinolaryngology and Head and Neck \\ Surgery, Kuwait \\ ${ }^{2}$ Department of ENT,AI Azhar Medical College and Super \\ Specialty Hospital, India
}

\begin{abstract}
Correspondence: Davis Thomas Pulimoottil, Department of ENT,Al Azhar Medical College and Super Specialty Hospital, Ezhalloor, Thodupuzha, Idukki Dist., Kerala - 685608, India, Tel +91 7639285181, Email davisthomasp@yahoo.in
\end{abstract}

Received: January 04, 2019 | Published: January 16, 2019

\section{Introduction}

A 42 year old female presented to the outpatient department with a slow growing painless mass in the left side of the floor of the mouth since 6 months which had been causing some difficulty in swallowing. On examination, the mass was non-tender, firm and noncompressible with well-defined borders, pushing the left side of the tongue up, and was not bidigitally palpable. It measured approximately $6 \times 4$ $\mathrm{cm}$ and was roughly oval in shape and there was no displacement or mobility of teeth noted. Intraoral examination revealed no reduced salivation from left Wharton's duct. Axial and coronal views of computed tomography (CT) imaging revealed a well-circumscribed soft tissue density mass in the left floor of mouth, separate from the deep lobe of the submandibular gland. The mass was excised under general anaesthesia and histopathological examination revealed darkly stained tumour cells lying in chondromyxoid mesenchyme, suggestive of pleomorphic adenoma of a minor salivary gland.

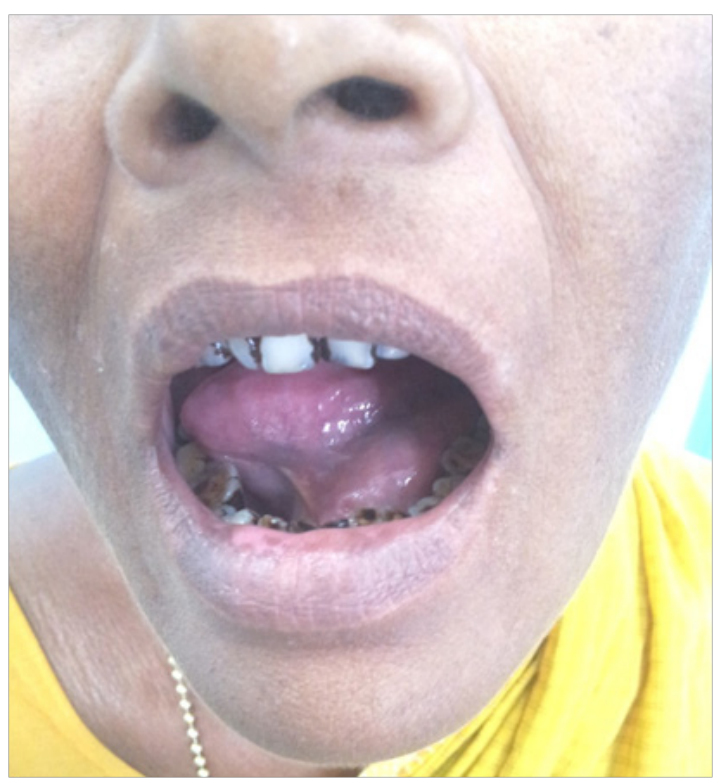

Figure I Clinical photograph showing a smooth well-defined mass on the left side of the floor of mouth elevating the tongue.
Pleomorphic adenomas, in general, comprise $40 \%$ of minor salivary gland tumors. Patients with pleomorphic adenomas of the minor salivary glands present mostly in their fourth to sixth decades, with a slight predominance in female patients. They usually present as a unilateral, painless, slow-growing mass in the parotid gland. ${ }^{1}$ However, when they originate in the minor salivary glands, they mostly occur in the hard and soft palate, the floor of mouth being a rarer site for the occurrence of these tumours, necessitating the need to differentiate from tumours of the submandibular gland. ${ }^{2}$ FNA biopsy should be performed as an adjunct to diagnosis prior to definitive surgical treatment. CT or magnetic resonance imaging (MRI) should be considered when assessing for presence of bony erosion or soft tissue and nerve involvement. ${ }^{3}$ Wide excision with negative margins is the optimal strategy for the management of pleomorphic adenomas. Recurrence may occur with inadequate initial surgical procedure (Figure 1) \& (Figure 2). ${ }^{4}$

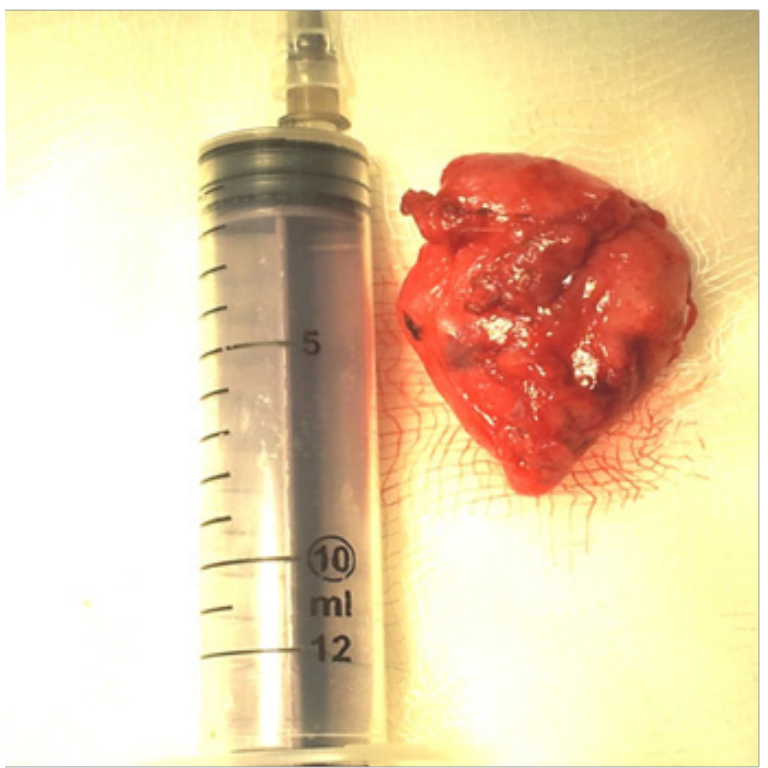

Figure 2 Gross specimen of the tumour post-excision revealing a wellencapsulated mass. 


\section{Funding details}

None.

\section{Acknowledgements}

None.

\section{Conflict of interest}

Authors declare that there is no conflict of interest.

\section{References}

1. Torske K. Benign neoplasm of the salivary glands. In: Thompson LDR (ed.) Head and neck pathology, 2006, 1st edn. Elsevier, Philadelphia, pp. 295-300.
2. Tian Z, Li L, Wang L, et al. Salivary gland neoplasms in oral and maxillofacial regions: A 23-year retrospective study of 6982 cases in an eastern Chinese population. Int J Oral Maxillofac Surg. 2009;39(3):235242 .

3. Wang D, Li Y, He H, et al. Intraoral minor salivary gland tumors in a Chinese population: A retrospective study on 737 cases. Oral Surg Oral Med Oral Pathol Oral Radiol Endod. 2007;104(1):94-100.

4. Mendenhall WM, Mendenhall CM, Werning JW, et al. Salivary gland pleomorphic adenoma. Am J Clin Oncol. 2008;31:95-99. 